\title{
Balanced Steady-State Free Precession Techniques Improve Detection of Residual Germ Cell Tumor for Treatment Planning
}

\author{
(D)W.A. Mehan, Jr, (D) K. Buch, (D) M.F. Brasz, (DF.F.J. Simonis, DS. MacDonald, (D). Rincon, (D).E. Kirsch, and (DP. Caruso
}

\begin{abstract}
BACKGROUND AND PURPOSE: Identification of a partial/complete chemotherapy response in pediatric patients with intracranial germ cell tumors is clinically important for radiation treatment and management. Partial/complete response is conventionally determined on postcontrast MR imaging sequences. The purpose of this study was to assess the diagnostic utility of a balanced steady-state free precession sequence as an adjunct to standard MR imaging sequences for the detection of residual tumor in pediatric patients on postchemoreduction pre-radiation planning MR imaging.
\end{abstract}

MATERIALS AND METHODS: This was a retrospective study of pediatric patients with intracranial germ cell tumors undergoing postchemotherapy, preradiotherapy MR imaging. Patients underwent 1.5T or 3T MR imaging with pre- and postcontrast TIWIs, T2WIs, and a balanced steady-state free precession sequence. Two neuroradiologists independently reviewed standard MR imaging sequences without the balanced steady-state free precession sequence, then with the balanced steady-state free precession sequence 1 week later. Assessment for partial/complete response was determined using Response Assessment in Neuro-Oncology criteria. A 5-point Likert scale scored the diagnostic confidence of the neuroradiologist rating each study without/with the balanced steady-state free precession sequence. Rates of residual disease concordance and diagnostic confidence levels without/with the balanced steady-state free precession sequence were calculated.

RESULTS: Thirty-nine patients were included with 31 males and 8 females (mean age, $14.15 \pm 4.26$ years). Thirty-one patients had single-site disease; 8 patients had multisynchronous disease (47 sites in total). Compared to review of the standard MR sequences alone, the addition of the balanced steady state free precession sequence resulted in higher rates of tumor partial response categorization and greater diagnostic confidence levels $(P<.001, P<.001)$.

CONCLUSIONS: The balanced steady-state free precession sequence improves detection of residual chemotherapy-reduced intracranial germ cell tumors and increases diagnostic confidence of the neuroradiologist. The balanced steady-state free precession sequence may be an important adjunct to the standard MR imaging protocol for radiation planning.

ABBREVIATIONS: BRAVO = axial MRI 3D brain volume; bSSFP = balanced steady-state free precession; $C R=$ complete response; GCT = germ cell tumor; $P R=$ partial response; $S P G R=$ spoiled gradient echo

$\mathrm{S}$ tandard of care treatment for CNS germ cell tumors (GCTs) is chemotherapy followed by radiation therapy. ${ }^{1}$ Treatment is based on the amount of residual tumor after chemotherapy. Patients with a complete response (CR) are defined by response assessment in neuro-oncology (RANO) criteria as having a complete radiographic disappearance of all tumor, whereas those with

Received December 12, 2019; accepted after revision March 16, 2020.

From the Departments of Radiology (W.A.M, Jr., K.B., S.R., J.E.K., P.C.) and Radiation Oncology (S.M.), Massachusetts General Hospital and Harvard Medical School, Boston, Massachusetts; and Magnetic Detection and Imaging (M.F.B., F.F.J.S.), TechMed Centre, University of Twente, Enschede, the Netherlands.

Please address correspondence to William A. Mehan, Jr, MD, MBA, Massachusetts General Hospital, 55 Fruit St, Gray 2-273A, Boston, MA 02114; e-mail: mehan.william@mgh.harvard.edu

http://dx.doi.org/10.3174/ajnr.A6540

a partial response (PR) are defined as having a $\geq 50 \%$ decrease (compared with baseline) in the sum of the products of the perpendicular diameters of the tumor. ${ }^{1,2} \mathrm{MR}$ imaging is considered the standard of care for assessment of residual tumor and thus is used to determine $\mathrm{CR}$ versus $\mathrm{PR}$.

The balanced steady-state free precession (bSSFP) technique is a heavily water-weighted $3 \mathrm{D}$ sequence used with isotropic or near-isotropic spatial encoding, which has been shown to be useful in neuro-oncologic imaging, given its high signal-to-noise ratio and superior discrimination of soft tissue from CSF within the ventricular system or cisterns. ${ }^{3-8}$ Recent consensus recommendations on MR imaging for the pediatric brain include axial T2-weighted images, axial diffusion-weighted images, and 3D T1 pre- and postcontrast sequences but do not specifically include bSSFP sequences. ${ }^{2}$ 
The assessment of CR versus PR is clinically relevant for the management and treatment of these patients. In patients with either CR or PR with residual disease of $<1 \mathrm{~cm}$, radiation therapy protocols typically include $18 \mathrm{~Gy}$ to the whole ventricles with a boost of 12 Gy to the primary tumor site. In patients with PR but with residual disease measuring between 1 and $1.5 \mathrm{~cm}$, a higher whole-ventricular radiation dose to $24 \mathrm{~Gy}$ with a $12-\mathrm{Gy}$ boost is delivered. Furthermore, if patients with a PR have residual disease measuring $>1.5 \mathrm{~cm}$, a second-look surgery is considered to assess for the presence of residual GCT such as mature teratoma versus scar. ${ }^{9-12}$

The purpose of our study was to evaluate whether the bSSFP sequence improves the detection of residual chemoreduced germ cell tumor over the standard neuro-oncologic MR imaging sequences.

\section{MATERIALS AND METHODS Study Design}

This retrospective, institutional review board-approved study included pediatric patients who presented to Massachusetts General Hospital between 2010 and 2019 with an intracranial GCT. Patients were identified through a search of the institutional radiology information system and an in-house clinical neuro-oncology data base. Inclusion criteria for this study were patients 21 years of age or younger; a clinical diagnosis of intracranial GCT based on biopsy or CSF; and paired prechemotherapy MR imaging examinations and postchemotherapy, preradiation MR imaging examinations. Standard sequences included pre- and postcontrast T1WI, diffusionweighted sequences, and T2WI as well as a bSSFP sequence. Patients with prior radiation treatment and nondiagnostic MRIs related to technical factors and motion artifacts were excluded.

\section{Clinical Data}

The electronic medical record for each patient was reviewed for the following: 1) demographic information, including age and sex; 2) tumor histology (eg, germinoma, yolk sac tumor, mature teratoma, or embryonal carcinoma); 3) tumor site (pineal, suprasellar, multiple midline, posterior fossa, purely intraventricular); and 4) treatment history, including a prior operation (date and biopsy versus debulking), chemotherapy (date and type), and radiation planning dose.

\section{Scanner Hardware and Sequence Parameters}

Imaging was performed on either a 1.5T (Signa Excite HDx, GE Healthcare; Avanto, Siemens) or 3T scanner (Discovery MR750, GE Healthcare; Tim Trio and Skyra, Siemens). The scans were obtained with an 8-channel brain array coil or a 16-channel head, neck, and spine coil for the Signa Excite HDx; a 20-channel head coil for the Avanto; a 16-channel head, neck, and spine coil for the Discovery MR750; and a 32-channel head coil for the Tim Trio.

\section{Imaging Protocol}

General anesthesia, child life specialists, and video goggles were used when deemed appropriate and based on our institutional protocols.
The standard acquired sequences obtained included a pregadolinium 3D T1WI, axial diffusion-weighted imaging, and axial T2WI. Following an intravenous bolus injection of gadoterate meglumine at $0.2 \mathrm{~mL} / \mathrm{kg}$ (Dotarem; Guerbet), a postcontrast 3D T1WI was performed. Scan parameters such as TE, TR, and flip angle were optimized per patient.

The scan parameters for the $1.5 \mathrm{~T}$ Avanto scanner for the T1WI (MPRAGE) pre- and postgadolinium were TR/TE $=2140$ / 4.75 to $2200 / 4.76 \mathrm{~ms}, \mathrm{NEX}=1$, matrix $=256 \times 256$, flip angle $=$ $15^{\circ}, \mathrm{FOV}=250 \times 250$ to $256 \times 256 \mathrm{~mm}$, section thickness $=$ $1 \mathrm{~mm}$, gap $=0 \mathrm{~mm}$, with an acquisition time of approximately 5 minutes, 30 seconds. For T2WI, the parameters were TR/TE = $3000 / 104$ to $5510 / 114 \mathrm{~ms}, \mathrm{NEX}=2$, matrix $=256 \times 256$ to $448 \times 448$, flip angle $=180^{\circ}$, FOV $=160 \times 160$ to $175 \times$ $220 \mathrm{~mm}$, section thickness $=3 \mathrm{~mm}$, gap $=3 \mathrm{~mm}$, with an acquisition time of approximately 4 minutes, 30 seconds.

The scan parameters for the GE 1.5T Signa Excite HDx scanners for the T1WI (axial MRI 3D brain volume [BRAVO; GE Healthcare] and echo-spoiled gradient echo [SPGR]; GE Healthcare) pre- and postgadolinium were TR/TE $=14.66 /$ $6.36 \mathrm{~ms}$ to $15.59 / 6.64 \mathrm{~ms}$ for BRAVO and $32 / 8 \mathrm{~ms}$ for SPGR, $\mathrm{NEX}=1$, matrix $=260 \times 260$ for BRAVO and $256 \times 192$ for SPGR, flip angle $=13^{\circ}$ for BRAVO and $25^{\circ}$ for SPGR, FOV $=$ $260 \times 260 \mathrm{~mm}$ for BRAVO and $220-340 \mathrm{~mm}$ for SPGR, section thickness $=1 \mathrm{~mm}$ for BRAVO and $1.5 \mathrm{~mm}$ for SPGR, gap $=1 \mathrm{~mm}$ for BRAVO and $1.5 \mathrm{~mm}$ for SPGR, with an acquisition time of approximately 3 minutes, 30 seconds for BRAVO and 5.9-7.5 minutes for SPGR. For the T2WI, the parameters were $\mathrm{TR} / \mathrm{TE}=4150 / 95.5$ to $6700 / 97.2 \mathrm{~ms}$, NEX $=$ 2 , matrix $=320 \times 256$, flip angle $=90^{\circ}, \mathrm{FOV}=220 \times 220$ to $240 \times 240 \mathrm{~mm}$, section thickness $=3 \mathrm{~mm}$, gap $=3 \mathrm{~mm}$, with an acquisition time of approximately $3-5$ minutes, 30 seconds.

The scan parameters for the 3T Siemens Tim Trio scanner and the 3T Skyra scanner for the T1WI (MPRAGE) pre- and postgadolinium were $\mathrm{TR} / \mathrm{TE}=2530 / 7.22 \mathrm{~ms}, \mathrm{NEX}=1$, matrix $=256 \times$ 256, flip angle $=7^{\circ}, \mathrm{FOV}=256 \times 256 \mathrm{~mm}$, section thickness $=$ $1 \mathrm{~mm}$, gap $=0 \mathrm{~mm}$, with an acquisition time of approximately 4-6 minutes, 18 seconds. For the T2WI, they were TR/TE $=3890 / 97$ to $8760 / 97 \mathrm{~ms}, \mathrm{NEX}=2$, matrix $=512 \times 294$ to $512 \times 336$, flip angle $=150^{\circ}, \mathrm{FOV}=256 \times 256 \mathrm{~mm}$, section thickness $=3 \mathrm{~mm}$, gap $=3 \mathrm{~mm}$, with an acquisition time of 3 minutes, 6 seconds -4 minutes, 30 seconds.

The scan parameters for the 3T Discovery MR750 scanner for the T1WI (SPGR) pre- and postgadolinium were TR/ $\mathrm{TE}=8.219 / 3.22 \mathrm{~ms}, \mathrm{NEX}=1$, matrix $=256 \times 256$, flip angle $=20^{\circ}, \mathrm{FOV}=240 \times 240 \mathrm{~mm}$, section thickness $=1.2 \mathrm{~mm}$, gap $=1.2 \mathrm{~mm}$, with an acquisition time of approximately 2 minutes, 12 seconds. For the T2WI, they were TR/TE $=4747$ / $84.8 \mathrm{~ms}, \mathrm{NEX}=2$, matrix $=384 \times 288$, flip angle $=111^{\circ}, \mathrm{FOV}=$ $220 \times 220 \mathrm{~mm}$, section thickness $=3 \mathrm{~mm}$, gap $=3 \mathrm{~mm}$, with an acquisition time of approximately 1 minute, 48 seconds.

The bSSFP sequence on the 3T Tim Trio and Skyra scanners (CISS) was performed using the following parameters: TR/TE $=$ $11.26 / 5.85$ to $12.55 / 6.28 \mathrm{~ms}, \mathrm{NEX}=1$, matrix $=448 \times 269$, flip angle $=50^{\circ}, \mathrm{FOV}=160 \times 160$ to $199 \times 199 \mathrm{~mm}$, section thickness $=0.8 \mathrm{~mm}$, gap $=0 \mathrm{~mm}$, with an acquisition time of approximately 8 minutes.

AJNR Am J Neuroradiol 41:898-903 May 2020 www.ajnr.org 
Table 1: Difference between the recordings with and without the bSSFP sequence for all cases and separately for different locations ${ }^{\mathrm{a}}$

${ }^{a}$ The recordings of the intraventricular and posterior fossa locations are described with the label "other location."

\begin{tabular}{|c|c|c|c|}
\hline \multicolumn{2}{|c|}{ Reader 1} & \multicolumn{2}{|c|}{ Reader 2} \\
\hline Without bSSFP & With bSSFP & Without bSSFP & With bSSFP \\
\hline \multicolumn{4}{|l|}{ All cases (47) } \\
\hline 28 CR $(60 \%)$ & 4 CR (9\%) & 22 CR (47\%) & 2 CR (4\%) \\
\hline 19 PR (40\%) & 43 PR (91\%) & 25 PR (53\%) & 45 PR $(96 \%)$ \\
\hline \multicolumn{4}{|l|}{ Pineal location (27) } \\
\hline 15 CR (56\%) & 3 CR (11\%) & 10 CR (37\%) & $1 \mathrm{CR}(4 \%)$ \\
\hline 12 PR (44\%) & 24 PR (89\%) & 17 PR $(63 \%)$ & $26 \operatorname{PR}(96 \%)$ \\
\hline \multicolumn{4}{|c|}{ Suprasellar location (17) } \\
\hline 12 CR $(71 \%)$ & $1 \mathrm{CR}(6 \%)$ & 11 CR (65\%) & 1 CR (6\%) \\
\hline 5 PR (29\%) & 16 PR (94\%) & 6 PR (35\%) & 16 PR (94\%) \\
\hline \multicolumn{4}{|l|}{ Other location (3) } \\
\hline 2 CR $(67 \%)$ & $0 \mathrm{CR}$ & 2 CR $(67 \%)$ & $0 \mathrm{CR}$ \\
\hline $1 \mathrm{PR}(33 \%)$ & 3 PR (100\%) & $1 \mathrm{PR}(33 \%)$ & 3 PR (100\%) \\
\hline
\end{tabular}

compared with the presentation MR imaging; stable disease, defined as no appreciable change in tumor volume; and progressive disease, defined as $\geq 25 \%$ increase in the sum of the products of perpendicular diameters. ${ }^{1}$

Cubic tumor volumes (anteroposterior $\times$ transverse $\times$ superoinferior) were measured on all prechemotherapy, pre-radiation therapy MRIs on the volumetric $\mathrm{T} 1$ postgadolinium and bSSFP sequences and on all postchemotherapy, pre-radiation therapy MRIs for which a PR was recorded. Cubic tumor volumes were calculated for all cases on both the volumetric T1 postgadolinium and bSSFP sequences.

The bSSFP sequence on the 3T Discovery MR750 scanners (FIESTA) was performed using the following parameters: TR/ $\mathrm{TE}=6.878 / 2.78 \mathrm{~ms}, \mathrm{NEX}=1$, matrix $=320 \times 256$, flip angle $=$ $65^{\circ}, \mathrm{FOV}=160 \times 160 \mathrm{~mm}$, section thickness $=1 \mathrm{~mm}$, gap $=$ $1 \mathrm{~mm}$, with an acquisition time of approximately 4 minutes, 6 seconds.

The bSSFP sequence on the 1.5T Avanto scanner (CISS) was performed using the following parameters: TR/TE $=5.72 / 2.4-$ $11.94 / 5.97 \mathrm{~ms}, \mathrm{NEX}=1$, matrix $=320 \times 224$, flip angle $=70-$ $80^{\circ}$, FOV $160 \times 160$ to $170 \times 170 \mathrm{~mm}$, slice thickness $=0.7-0.8$ $\mathrm{mm}$, gap $=0 \mathrm{~mm}$, with an acquisition time of approximately 5 minutes, 24 seconds.

The bSSFP sequence on the 1.5T Signa Excite HDx scanner (FIESTA) was performed using the following parameters: TR/ $\mathrm{TE}=6.048 / 2.33$ to $12.47 / 2.43 \mathrm{~ms}, \mathrm{NEX}=1-6$, matrix $=448 \times$ 256, flip angle $=65^{\circ}$, FOV $=180 \times 180$ to $220 \times 220 \mathrm{~mm}$, section thickness $=0.8-1 \mathrm{~mm}$, gap $=0.4-0.5 \mathrm{~mm}$, with an acquisition time of approximately 2 minutes, 54 seconds -8 minutes, 54 seconds.

\section{Image Analysis}

The presentation, pretreatment MRIs and the postchemotherapy, preradiation MRIs were reviewed by 2 neuroradiologists (W.A.M. with $>5$ years' postfellowship experience and P.C. with $>15$ years' postfellowship experience), independently, at 2 separate sessions. During the first session, informed only of the location of the tumor, the 2 neuroradiologists reviewed the cases with standard neuro-oncologic sequences that included axial T2WI and postgadolinium SPGR/MPRAGE/ BRAVO sequences, but without the bSSFP sequence. During the second session, at least 1 week later, the same 2 neuroradiologists independently reviewed all sequences, but this time with the addition of the bSSFP sequence. An interval of at least 1 week was chosen between the first and second readout sessions to avoid memory bias.

At both the first and second readout sessions, the 2 neuroradiologists evaluated the primary treatment bed as per Kretschmar et al for CR, defined as no radiographic evidence of disease at the site of the original tumor; $\mathrm{PR}$, defined as $\geq 50 \%$ decrease in the sum of the products of perpendicular diameters of the lesion
A 5-point Likert scale confidence score $(1=$ not confident at all, $2=$ slightly confident, $3=$ somewhat confident, $4=$ fairly confident, $5=$ completely confident) was recorded for each neuroradiologist's assessment of CR versus PR during both review sessions without-versus-with the inclusion of the bSSFP sequence. ${ }^{12}$

\section{Statistical Analysis}

A 2-sample test of proportions and a Fisher exact test were performed to analyze differences in confidence scores and assessment of CR versus PR in the bSSFP and non-bSSFP groups. The $\kappa$ score was used to assess the level of interrater agreement between the 2 radiologists with SPSS statistics 25 (IBM).

\section{RESULTS}

\section{Cohort Description}

A total of 74 patients were considered for this study. Thirty-five patients were excluded, with 31 due to lack of a bSSFP sequence, 2 due to prior radiation therapy, and 2 due to motion artifacts. In this cohort, a total of 39 pediatric patients were included, 31 males and 8 females, ranging from 4 to 21 years of age (mean, $14.15 \pm 4.26$ years). Twenty-four patients were diagnosed with a pure germinoma, and 15 patients were diagnosed with a nongerminomatous germ cell tumor. All patients had documented negative serum and CSF tumor markers before evaluation for radiation therapy. The nongerminomatous germ cell tumor group contained 1 yolk sac tumor, 1 mature teratoma, 1 embryonal carcinoma, and 12 mixed tumors with germ cell and mature/immature or yolk sac components. Of the 39 patients, 19 had pineal tumor location only, 9 had suprasellar tumor location only, 2 had intraventricular location only, 1 had posterior fossa location only and 8 patients had multiple midline germ cell tumors, including the suprasellar and pineal regions. There were 47 tumor locations in total: Twenty-seven tumors were pineal, 17 tumors were suprasellar, 2 tumors were intraventricular, and 1 tumor was located in the posterior fossa.

\section{Imaging Protocol}

Twenty-seven patients were scanned on a $1.5 \mathrm{~T}$ scanner, and 12 patients were scanned on a $3 \mathrm{~T}$ scanner. 


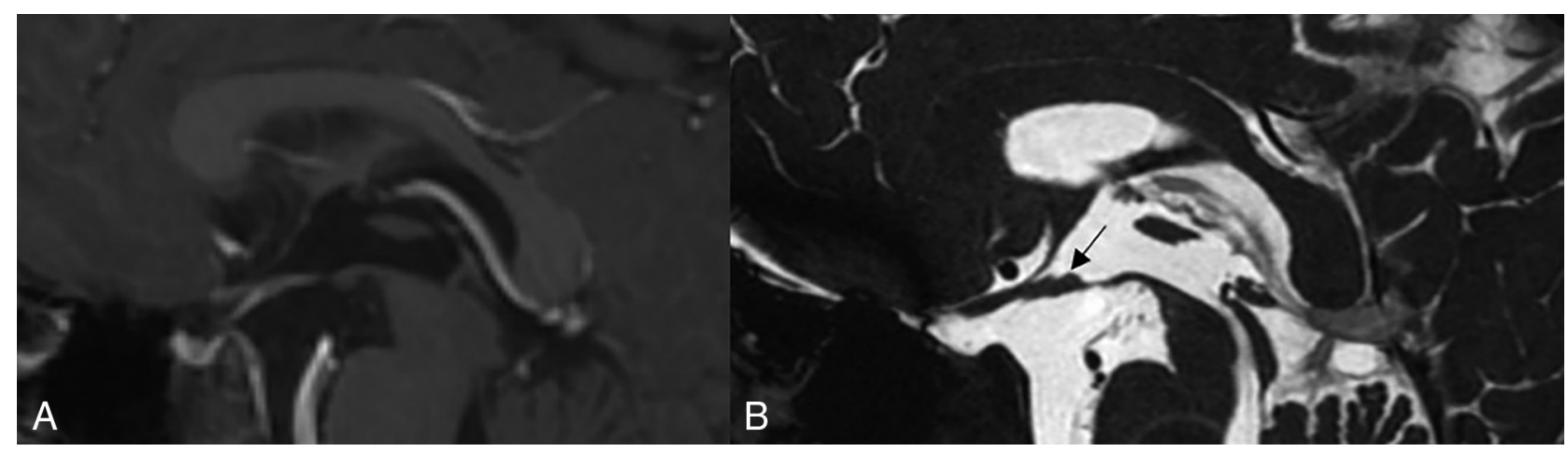

FIG 1. A 10-year-old boy with a history of chemotherapy-treated suprasellar germ cell tumor. Sagittal 3D T1 postcontrast SPGR image through the sella $(A)$, interpreted as complete response in the first readout session without the bSSFP sequence. Sagittal FIESTA image through the sella $(B)$ from the same patient interpreted as partial response during the second readout session with small nodules identified along the floor of the third ventricle (arrow).

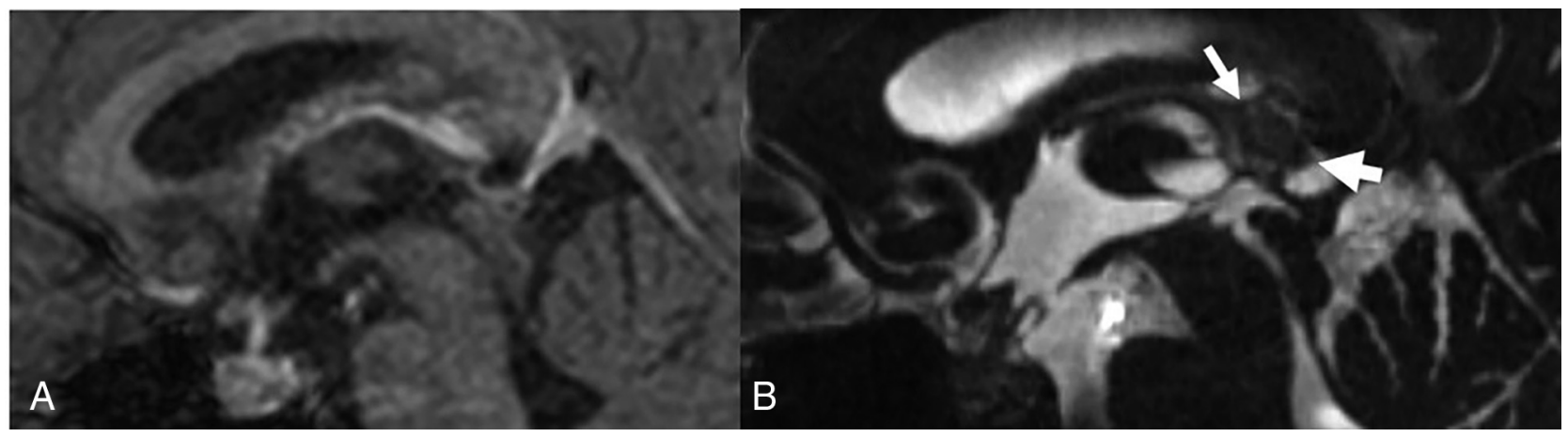

FIG 2. A 19-year-old woman with a history of chemotherapy-treated pineal germ cell tumor. Sagittal 3D T1 postcontrast MPRAGE image through pineal region $(A)$ interpreted as complete response in the first readout session without the bSSFP sequence. Sagittal CISS image through the pineal region $(B)$ from the same patient interpreted as partial response during the second readout session with a $>1-\mathrm{cm}$ nodule identified within the pineal gland (arrows).

\section{Evaluation of $C R$ and $P R$}

Evaluation of Response during First Review (without the bSSFP). Reader 1 detected 28 instances of CR, 19 PR, 0 stable disease, and 0 progressive disease during the first review in all cases, and reader 2 recorded $22 \mathrm{CR}, 25 \mathrm{PR}, 0$ stable disease, and 0 progressive disease.

Evaluation of Response during Second Review (with the bSSFP). During the second review, with the addition of the bSSFP sequence, reader 1 recorded $4 \mathrm{CR}, 43 \mathrm{PR}, 0$ stable disease, and 0 progressive disease in all cases. Reader 2 recorded 2 CR, 45 PR, 0 stable disease, and 0 progressive disease.

Table 1 shows the difference between the recordings with and without the bSSFP sequence for all cases and separately for different locations.

There were 24 cases (reader 1) and 20 cases (reader 2) that were initially interpreted as CR during the first session of the evaluation and were recategorized as PR during the second review with the addition of the bSSFP sequence (Figs 1 and 2). There was a significant difference between the number of $C R$ and $\mathrm{PR}$ evaluations between the recordings with and without the bSSFP sequence $(P<.001$, Fisher exact test performed for both readers).

\section{Tumor Size and Volume Measurements}

For the presentation MRIs, the mean cubic tumor volume on the volumetric postcontrast T1WI was $8.88 \pm 12.85 \mathrm{~cm}^{3}$ (range, $0.06-$ $68.94 \mathrm{~cm}^{3}$ ). The mean cubic tumor volume for all PR evaluations on the volumetric postcontrast T1WI was $0.97 \pm 1.67 \mathrm{~cm}^{3}$ (range, $0.004-7.54 \mathrm{~cm}^{3}$ ). The mean cubic tumor volume for the PR evaluations on the bSSFP sequence was $1.31 \pm 1.88 \mathrm{~cm}^{3}$ (range, 0.016$8.09 \mathrm{~cm}^{3}$ ). Although the mean cubic tumor volume on the postchemotherapy preradiation planning MRIs was slightly higher on the bSSFP sequence compared with the postcontrast T1WI, the difference was not significant $(P=.33)$.

Of the cases recategorized from CR to PR after review of the bSSFP sequence, the mean tumor volume was $0.24 \pm 0.55 \mathrm{~cm}^{3}$ (range, $0.002-2.84 \mathrm{~cm}^{3}$ ), and 1 of these cases had a volume of $>1 \mathrm{~cm}^{3}$.

Of the cases recategorized from CR to PR after review of the bSSFP sequence, 6 tumors had a single plane measurement of $\geq 1$ $\mathrm{cm}$ (average single plane measurement, $1.4 \pm 3.2 \mathrm{~cm}$ ).

\section{Confidence Scores}

The confidence scores of both sessions and the difference between the interval per location are shown in Table 2. Reader 1 noted a confidence interval of $2-5$ (mean, $4.17 \pm 0.82$ ) for all cases during 
Table 2: Confidence scores recorded by the 2 readers for all cases and for individual locations on the bSSFP sequence compared with postcontrast $\mathrm{TIWI}^{\mathrm{a}}$

\begin{tabular}{lcc}
\hline & Reader 1 & Reader 2 \\
\hline All cases (47) & $4.17 \pm 0.82(2-5)$ & $4.36 \pm 0.61(3-5)$ \\
TIWI & $4.77 \pm 0.43(4-5)$ & $4.55 \pm 0.58(3-5)$ \\
bSSFP & & \\
Pineal location (27) & $4.07 \pm 0.96(2-5)$ & $4.41 \pm 0.67(3-5)$ \\
$\quad$ Without bSSFP & $4.74 \pm 0.45(4-5)$ & $4.63 \pm 0.56(3-5)$ \\
$\quad$ With bSSFP & \\
Suprasellar location (17) & $4.35 \pm 0.61(3-5)$ & $4.29 \pm 0.69(3-5)$ \\
$\quad$ Without bSSFP & $4.94 \pm 0.24(4-5)$ & $4.47 \pm 0.28(3-5)$ \\
$\quad$ With bSSFP & & \\
Other location (3) & $4 \pm 0.00(4)$ & $4.3 \pm 0.58(4-5)$ \\
$\quad$ Without bSSFP & $4 \pm 0.00(4)$ & $4.3 \pm 0.58(4-5)$ \\
$\quad$ With bSSFP & & \\
\hline a Data are mean and range. &
\end{tabular}

the first recording. Reader 2 noted a confidence interval of 3-5 (mean, $4.36 \pm 0.61$ ).

During the second review session, with the bSSFP sequence, the mean confidence score for both readers was higher. Reader 1 showed a confidence interval of $4-5$ (mean, $4.77 \pm 0.43$ ) for all cases. Reader 2 noted a confidence interval of 3-5 (mean, $4.55+0.58)$.

There was a statistically significant difference between the confidence scores between the reviews performed with the bSSFP sequence compared with reviews performed without bSSFP sequence $(P<.001,2$-tailed $t$ test $)$.

\section{Interrater Reliability}

The number of discrepant cases between readers 1 and 2 during the first review, without the bSSFP sequence, was 12 . The number of discrepancies between readers 1 and 2 during the second review, with the bSSFP sequence, was 4 . For the first review session, without the bSSFP, the calculated $\kappa$ score between reader 1 and reader 2 was 0.128 . For the second review session, with the bSSFP, the calculated $\kappa$ score between reader 1 and 2 was 0.872 .

\section{DISCUSSION}

Our study demonstrates that the addition of a bSSFP technique to a neuro-oncology MR imaging protocol increases the detection of residual germ cell tumor and improves the interpreting neuroradiologist's confidence in reporting the presence of residual disease.

Compared with the neuroimaging standard of T1-weighted postcontrast imaging for assessing tumor response, the bSSFP sequence adds value through improved contrast and higher spatial resolution, which facilitates the detection of nonenhancing or minimally enhancing tumor components. These features allow improved residual germ cell tumor detection, particularly in the typical locations in the suprasellar and pineal regions, which are surrounded by CSF, thus allowing superior contrast resolution between hypointense tumor and hyperintense CSF.

The sensitivity of bSSFP sequences for the detection of small volumes of residual tumor is exemplified by the 24 (reader 1 ) and 20 (reader 2) cases reclassified from CR to PR by the addition of bSSFP sequences. The residual tumor volumes were, on average, $0.25 \mathrm{~cm}^{3}$, which are small and could be potentially overlooked on standard T1 postcontrast and T2/FLAIR sequences. A total of 6 cases had tumors that demonstrated a residual maximal singleplane measurement of $\geq 1 \mathrm{~cm}$, which, based on the Children's Oncology Group ACNS1123 protocol, would have stratified 4 patients for a radiation boost and 2 patients for second-look surgery. ${ }^{9-12}$ Another important finding is that the addition of a bSSFP sequence improves reader confidence in detecting residual tumor and determining PR-versus-CR status. The average confidence score for the 2 neuroradiologists was 4.17 with the standard MR images and 4.77 with the addition of the bSSFP sequence $(P<.001)$. The number of discrepant interpretations decreased from 12 with the standard MR images to 4 with the inclusion of the bSSFP sequence. Furthermore, interrater agreement also improved from poor (0.128) to excellent (0.872) with the bSSFP sequence.

Both the improved sensitivity for residual tumor and higher reader confidence with the bSSFP sequence when interpreting postchemotherapy-induction MR imaging studies are of paramount importance for therapeutic decision-making. Recategorizing patients from $\mathrm{CR}$ to $\mathrm{PR}$ is clinically relevant and may influence radiation therapy dosing and treated tumor volumes as well as help determine whether a second-look surgery may be necessary. ${ }^{1,2,9-12}$

Our findings are similar to those in other previous studies that have demonstrated the benefits of adding bSSFP sequences to standard neuroimaging protocols for CNS tumor detection, including high accuracy for vestibular schwannoma detection and spinal drop metastases. ${ }^{4,13-16}$

Although the inclusion of a bSSFP sequence increased the total scan length by 6 minutes on average, we found that the improved sensitivity and confidence in the interpretation justify the additional imaging time. Furthermore, there is diagnostic synergy between the bSSFP and the standard MR images when interpreted in conjunction. At our institution, for several years now, we have routinely performed a bSSFP sequence as part of the neuro-oncology MR imaging protocol, given that the pediatric neuroradiologists prefer this sequence for its high spatial resolution, isotropic images, and ability to characterize cystic tumoral components. One pitfall of bSSFP, however, is a banding artifact caused by destructive interference between the 2 primary coherent signal pathways in off-resonance regions of $\mathrm{B}_{0}$ inhomogeneities. This generates dark lines on the images that may at times obscure anatomic structures and lesions. ${ }^{17}$ CISS is a 2-pass bSSFP technique that attempts to minimize these artifacts. Nevertheless, in 1 case in our sample, there were a large amount of banding artifacts obscuring the pineal region on the bSSFP sequence; this issue was surmounted by simultaneously correlating with the postcontrast T1-weighted images.

This study has several limitations. The sample size was relatively small. Also, patients were imaged on different MR imaging scanners, which introduced heterogeneity between magnet field strengths (1.5T versus $3 \mathrm{~T}$ ) as well as between the vendor-specific bSSFP sequences (CISS for Siemens versus FIESTA for GE Healthcare). However, each patient's standard MR images and bSSFP sequence were performed on the same scanner. In no case was a comparison made in the same patient between MR images obtained at different magnetic field strengths or between different vendors. The increased rate of detection of tissue nodularity at the primary tumor site with the addition of the bSSFP sequence may not be clinically important in all patients, particularly given 
that most residual tumors detected were $<1 \mathrm{~cm}$. Given the investigational nature of our current study, additional studies are needed to determine whether these areas of presumed residual tumor identified on bSSFP imaging impact patient management and outcomes. Furthermore, there was a lack of histopathologic confirmation in most cases. Postsurgical and postchemotherapy scar tissue may have been misinterpreted as residual tumor, thus resulting in false-positives. However, histopathologic confirmation is not standard of care.

\section{CONCLUSIONS}

Adding a bSSFP sequence to the standard neuro-oncology MR imaging protocol improves the neuroradiologist's detection of presumed residual chemotherapy-reduced CNS germ cell tumor and increases confidence in distinguishing partial response from complete response disease status. This distinction could have important therapeutic consequences for the patient with regard to determining the radiation dose or the need for an additional operation; however, further investigations are required.

Disclosures: William A. Mehan—UNRELATED: Consultancy: Kura Oncology, Comments: reviewer of radiologic studies for a clinical trial dealing with head and neck cancer; Expert Testimony: CRICO and other medical insurance companies, Comments: expert review for malpractice cases involving neuroimaging.

\section{REFERENCES}

1. Kretschmar C, Kleinberg L, Greenberg M, et al. Pre-radiation chemotherapy with response-based radiation therapy in children with central nervous system germ cell tumors: a report from the Children's Oncology Group. Pediatr Blood Cancer 2007;48:285-91 CrossRef Medline

2. Warren KE, Vezina G, Poussaint TY, et al. Response assessment in medulloblastoma and leptomeningeal seeding tumors: recommendations from the Response Assessment in Pediatric Neuro-Oncology committee. Neuro Oncol 2018;20:13-23 CrossRef Medline

3. Hingwala D, Chatterjee S, Kesavadas C, et al. Applications of 3D CISS sequence for problem solving in neuroimaging. Indian J Radiol Imaging 2011;21:90-97 CrossRef Medline

4. Buch K, Caruso P, Ebb D, et al. Balanced steady-state free precession sequence (CISS/FIESTA/3D driven equilibrium radiofrequency reset pulse) increases the diagnostic yield for spinal drop metastases in children with brain tumors. AJNR Am J Neuroradiol 2018;39:1355-61 CrossRef Medline
5. Gloor M, Scheffler F, Bieri O. Quantitative magnetization transfer imaging using balanced SSFP. Magn Reson Med 2008;60:691-700 CrossRef Medline

6. Sheth S, Branstetter BF 4th, Escott EJ. Appearance of normal cranial nerves on steady-state free precession MR images. Radiographics 2009;29:1045-55 CrossRef Medline

7. Algin O, Hakyemez B, Gokalp G, et al. The contribution of 3D-CISS and contrast-enhanced MR cisternography in detecting cerebrospinal fluid leak in patients with rhinorrhea. Br J Radiol 2010;83:22532 CrossRef Medline

8. Algin O, Hakyemez B, Parlak M. Phase-contrast MRI and 3D-CISS versus contrast-enhanced MR cisternography on the evaluation of the aqueductal stenosis. Neuroradiology 2010;52:99-108 CrossRef Medline

9. Children's Oncology Group. ACNS1123: Phase 2 trial of responsebased radiation therapy for patients with localized central nervous system germ cell tumor. https://childrensoncologygroup.org/index. php/acns1123. Accessed February 20, 2020

10. Khatua S, Fangusaro J, Dhall G, et al. GC-17: the Children's Oncology Group (COG) current treatment approach for children with newly diagnosed central nervous system (CNS) localized germinoma (ACNS1123 STRATUM 2). Neuro Oncol 2016;18(Suppl 3): iii45.4-46 CrossRef

11. Osorio DS, Allen JC. Management of CNS germinoma. CNS Oncol 2015;4;273-79 CrossRef Medline

12. Murphy ES, Fangusaro J, Dhall G, et al. ACNS1123: Phase 2 trial of response-based radiation therapy for patients with localized central nervous system germ cell tumor (CNS GCT) - a Children's Oncology Group (COG) study. Patterns of failure and radiation dosimetry for nongerminomatous germ cell tumors. International Journal of Radiation Oncology Biology Physics 2018;102:S49-50 CrossRef

13. Abele TA, Besachio DA, Quigley EP, et al. Diagnostic accuracy of screening MR imaging using unenhanced axial CISS and coronal T2WI for detection of small internal auditory canal lesions. AJNR Am J Neuroradiol 2014;35:2366-70 CrossRef Medline

14. Stuckey SL, Harris AJ, Mannolini SM. Detection of acoustic schwannoma: use of constructive interference in the steady state threedimensional MR. AJNR Am J Neuroradiol 1996;17:1219-25 Medline

15. Hermans R, Van der Goten A, De Foer B, et al. MRI screening for acoustic neuroma without gadolinium: value of 3DFT-CISS sequence. Neuroradiology 1997;39:593-98 CrossRef Medline

16. Buch K, Juliano A, Stankovic KM, et al. Noncontrast vestibular schwannoma surveillance imaging including an MR cisternographic sequence: is there a need for postcontrast imaging? J Neurosurg 2018;131:549-54 CrossRef Medline

17. Bangerter NK, Hargreaves BA, Vasanawala SS, et al. Analysis of multiple-acquisition SSFP. Magn Reson Med 2004;51:1038-47 CrossRef Medline 